Revue d'histoire de l'Amérique française

REVUE D.HISTOIRE DE L'AMÉRIQUE FRANÇAISE

\title{
Le haut marais de l'Isle-aux-Grues : un exemple d'exploitation et de développement durables
}

\section{Catherine Plante, Matthew Hatvany et Najat Bhiry}

Volume 60, numéro 1-2, été-automne 2006

L'histoire environnementale

URI : https://id.erudit.org/iderudit/014594ar

DOI : https://doi.org/10.7202/014594ar

Aller au sommaire du numéro

\section{Éditeur(s)}

Institut d'histoire de l'Amérique française

ISSN

0035-2357 (imprimé)

1492-1383 (numérique)

Découvrir la revue

Citer cet article

Plante, C., Hatvany, M. \& Bhiry, N. (2006). Le haut marais de l'Isle-aux-Grues : un exemple d'exploitation et de développement durables. Revue d'histoire de l'Amérique française, 60(1-2), 37-60. https://doi.org/10.7202/014594ar
Résumé de l'article

Synonymes de richesses animales et végétales, les marais de l'estuaire du fleuve Saint-Laurent ont été exploités depuis longtemps, d'abord par les Amérindiens puis par les colons européens. Toutefois, l'industrialisation a entraîné leur destruction : plus de la moitié des milieux humides de l'estuaire du Saint-Laurent a disparu. Dans l'optique du développement durable, cet article a pour but d'explorer la relation géohistorique entre société et environnement sur le haut marais de l'Isle-aux-Grues, une relation qui a pris appui sur les limites et les processus naturels de l'écosystème, à la différence de ce qu'on observe dans plusieurs autres environnements humides de l'estuaire. Depuis quatre siècles, les humains ont développé un rapport durable avec la nature, en faisant du haut marais de l'Isle-aux-Grues un lieu de chasse, de pêche, de récolte du foin de mer et de loisir.
Tous droits réservés @ Institut d'histoire de l'Amérique française, 2006
Ce document est protégé par la loi sur le droit d'auteur. L'utilisation des services d’Érudit (y compris la reproduction) est assujettie à sa politique d'utilisation que vous pouvez consulter en ligne.

https://apropos.erudit.org/fr/usagers/politique-dutilisation/ 


\title{
Le haut marais de l'Isle-aux-Grues : un exemple d'exploitation et de développement durables
}

\author{
Catherine Plante \\ MatTHEW HatVany \\ NaJAT BHIRY \\ Département de géographie \\ Université Laval
}

RÉSUMÉ - Synonymes de richesses animales et végétales, les marais de l'estuaire du fleuve Saint-Laurent ont été exploités depuis longtemps, d'abord par les Amérindiens puis par les colons européens. Toutefois, l'industrialisation a entraîné leur destruction: plus de la moitié des milieux humides de l'estuaire du Saint-Laurent a disparu. Dans l'optique du développement durable, cet article a pour but d'explorer la relation géohistorique entre société et environnement sur le haut marais de l'Isle-aux-Grues ${ }^{1}$, une relation qui a pris appui sur les limites et les processus naturels de l'écosystème, à la différence de ce qu'on observe dans plusieurs autres environnements humides de l'estuaire. Depuis quatre siècles, les humains ont développé un rapport durable avec la nature, en faisant du haut marais de l'Isle-auxGrues un lieu de chasse, de pêche, de récolte du foin de mer et de loisir.

ABSTRACT - Synonymous with faunal and floral abundance, the marshes of the St. Lawrence Estuary have been exploited for centuries, first by Amerindians, then by European settlers. Industrialisation, however, brought about their destruction: more than half of the estuary's wetlands have disappeared. Within the context of sustainable development, this article explores the geohistorical relationship between society and the environment in the high marsh area of Isle-aux-Grues, a relationship that respects the natural processes and limits

1. Même si les règles de toponymie veulent que l'entité géographique s'écrive "îles aux Grues», le village «L'Isle-aux-Grues » et la paroisse «Saint-Antoine-de-l'Isle-aux-Grues», l'orthographe «Isle-aux-Grues» (et celui de "Isle-aux-Oies») a été choisi pour éviter la confusion. 
of this ecosystem, unlike what can be observed in many other wetlands in the estuary. Over the past four centuries, humans have developed a sustainable relationship with nature by using the high marsh area of Isle-aux-Grues for hunting, fishing, harvesting marsh hay and leisure activities.

\section{INTRODUCTION}

Dans des textes publiés au printemps 2005 dans le quotidien Le Devoir, le chroniqueur environnemental Louis-Gilles Francœur écrivait que «Le saccage des milieux humides a commencé au Québec avec la colonisation. [...] On considère trop souvent au Québec marais et marécages comme des terrains perdus ${ }^{2}$ ». «[D]es exemples récents montrent où les Québécois placent les milieux humides dans leur échelle de valeurs: des trous à bibittes qu'il vaut mieux remblayer ou assécher ${ }^{3}$.» En quelques lignes, il réduisait ainsi quatre cents ans de relations humaines avec les terres humides de la vallée du Saint-Laurent à une simple histoire de méconnaissance et de destruction d'un milieu naturel vital à la santé de la biosphère.

Ni terre ni mer, les marais intertidaux du Saint-Laurent ont toujours été difficiles à comprendre. Des naturalistes ont mené quelques recherches sur les marais dans les années 1930, mais l'étude écologique de ces milieux n'a commencé sérieusement qu'au début des années 1970. Rien d'étonnant, alors, à ce que la compréhension historique des relations humaines avec les milieux humides demeure aussi rudimentaire. Cependant, il faut reconnaître que de tout temps, en Europe comme en Amérique, les sources historiques laissent entrevoir deux façades des marais intertidaux. Plus souvent qu'autrement, les observateurs étrangers (visiteurs, membres du gouvernement, agronomes, scientifiques et aménagistes), percevaient les marais comme des lieux insalubres, pleins d'insectes et de maladies, et sans valeur. Par contre, ceux qui les fréquentaient sur une base quotidienne y voyaient des lieux de subsistance, de même que des pourvoyeurs de richesses écologiques et économiques (chasse, pêche, pâturage, etc.).

2. Louis-Gilles Francœur, «Mulcair s'engage à protéger tous les milieux humides : un amendement à la loi promis pour 2006", Le Devoir, 11 mai 2005.

3. Lous-Gilles Francour, «Milieux humides: une protection urgente: les orientations du gouvernement Charest sont accueillies favorablement par les groupes environnementaux", Le Devoir, 14 et 15 mai 2006. 
En insistant sur le caractère simple et linéaire des relations humaines avec les terres humides du Saint-Laurent, des interprétations comme celle offertes par Francœur et d'autres analystes ${ }^{4}$ ne sont pas sans rappeler ces discours venus de l'extérieur. Comme des géohistoriens l'ont illustré depuis plus d'un siècle, les relations humaines avec l'environnement sont beaucoup plus profondes et complexes. Faites de continuités et de discontinuités, parfois de ruptures, elles sont le résultat de processus qui impliquent à la fois des variables spatiales, temporelles, environnementales, économiques et culturelles ${ }^{5}$. Comme les êtres humains modifient leur environnement depuis des siècles afin de répondre à leurs besoins de survie, il importe de se questionner sur la place historique qu'ils occupent dans la nature. Comment intègrent-ils leur mode de vie à leur environnement immédiat? Leurs activités quotidiennes ont-elles un impact sur l'évolution de l'environnement? Pourraient-ils en utiliser les ressources naturelles sans causer de dommages importants? Les réponses à ces questions et à d'autres aideront à mieux connaître le rapport entre la société et l'environnement et, ainsi, à favoriser de meilleures pratiques d'utilisation du territoire en ce début de $\mathrm{xxI}^{\mathrm{e}}$ siècle. À cet égard, les marais du Saint-Laurent sont un milieu écologique tout indiqué pour nourrir notre réflexion sur l'impact historique de l'activité humaine sur l'environnement. Comme l'étude du haut marais de l'Isle-aux-Grues l'indique, chaque milieu et chaque communauté entretiennent des relations profondes, complexes et spécifiques qui doivent être analysées à la fois dans le temps et dans l'espace pour en dresser les particularités et l'histoire environnementale.

\section{LE LIEU D'ÉTUDE : LÀ OÙ POUSSE LE FOIN DE MER}

Au large de Montmagny, à 65 kilomètres en aval de Québec, le fleuve SaintLaurent abrite l'archipel de l'Isle-aux-Grues. Parmi les îles de cet archipel, l'Isle-aux-Grues, habitée par environ 120 personnes, est reliée à l'Isle-auxOies (située plus à l'est) par une bande de terre appelée «batture», qui est en réalité l'un des plus grands marais intertidaux de l'estuaire du Saint-

4. Jean Gauthier, Denis Lehoux et Jacques Rosa, Les marécages intertidaux dans l'estuaire du Saint-Laurent (Ottawa, Environnement Canada, Service canadien de la faune, 1980), 1; Ursula Larouche, «Les milieux humides: attention fragiles!», Continuité, 64 (printemps 1995): 26-27.

5. Vidal de la Blache, Principes de géographie humaine (Paris, Armand Colin, $5^{\mathrm{e}}$ édition, 1955); Carl Sauer, "The Morphology of Landscape», University of California Publications in Geography, 2 (1925): 19-54; Michael Williams, "The Relations of Environmental History and Historical Geography", Journal of Historical Geography, 20, 1 (1994): 3-21. 
Laurent. La batture de l'Isle-aux-Grues est formée de sédiments argileux et de matière organique emprisonnés entre des affleurements rocheux appartenant aux Appalaches. Exploité continuellement depuis plus de mille ans, cet écosystème humide a une grande valeur écologique et même économique ${ }^{6}$ (figures 1 et 2 ).

\section{Figure I}

Localisation de quelques marais de l'estuaire du Saint-Laurent

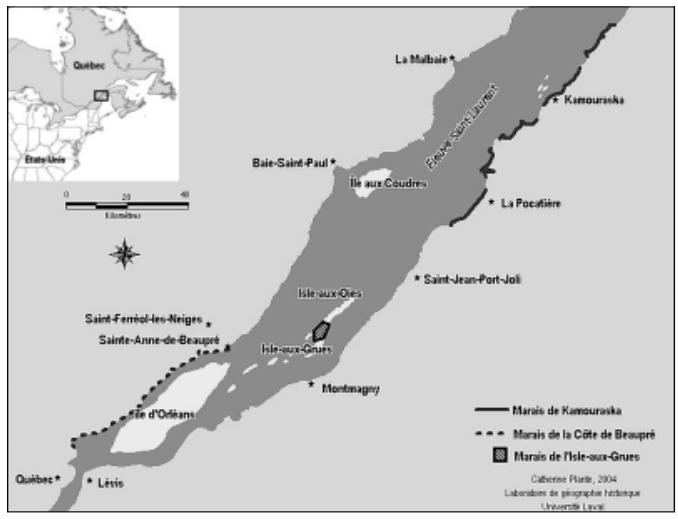

Source: Plante, 2005,8.

Figure 2

Les marais de l'Isle-aux-Grues et de l'Isle-aux-Oies

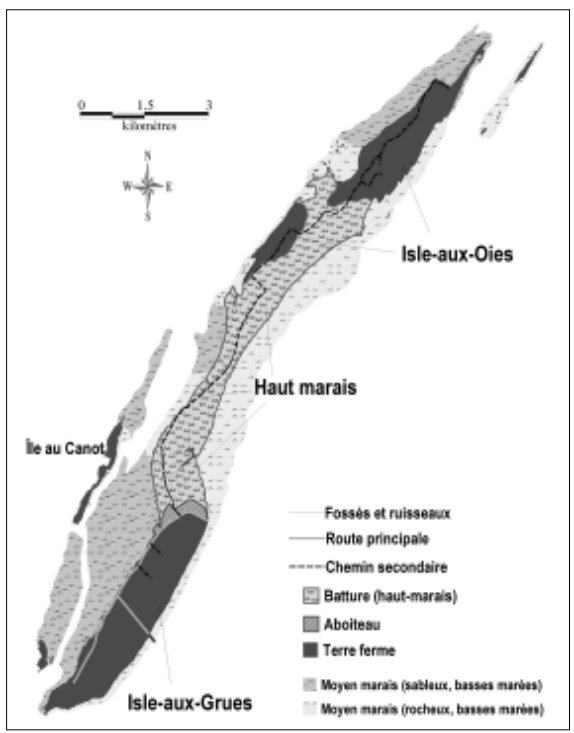

Source: Plante, 2005, 24. 
Le haut marais de l'Isle-aux-Grues est caractérisé par un sol toujours humide en raison de l'influence des marées quotidiennes, lesquelles remplissent le réseau hydrographique (ruisseaux, canaux de drainage et fossés) parsemant le marais. Ce dernier, ainsi que le chemin reliant les deux îles et passant sur le marais, sont recouverts sporadiquement par les grandes marées, dont celles d'équinoxes et des périodes de tempêtes. La surface du marais est plane, assez uniforme et d'une superficie d'environ $14 \mathrm{~km}^{2}$. Malgré une présence humaine très ancienne, cet environnement humide donne l'impression d'une grande plaine sauvage couverte d'une végétation très haute, dense et variée, appelée autrefois «foin de mer». Cette expression rassemble des espèces végétales très diverses (carex, spartine, joncs et scirpes), récoltées pour nourrir les troupeaux de bovins et autres animaux de ferme et domestiques.

Les marais de l'estuaire du Saint-Laurent comptent parmi les écosystèmes les plus productifs en biomasse (ou matière organique) au Québec, soit jusqu'à deux fois plus que les meilleures terres agricoles. Ces milieux, caractérisés par une richesse écologique exceptionnelle bien reconnue par les Amérindiens et les premiers colons, sont très vulnérables à l'action anthropique. Comme plusieurs auteurs l'ont montré, dont Teal et Teal, Gauthier et al. et Hatvany, voilà 150 ans, les marais de l'estuaire du SaintLaurent ont, depuis les débuts de l'industrialisation en Amérique du Nord, été perçus comme des lieux malodorants et sources de maladies. Jugés sans valeur, ces écosystèmes ont été endigués pour augmenter les superficies agricoles et remblayés pour construire des infrastructures d'urbanisation (autoroutes, stationnements et bâtiments). Au total, ce sont ainsi de $50 \%$ à $80 \%$ des milieux humides de l'estuaire du Saint-Laurent qui ont disparu depuis $1850^{7}$. Cependant, le cas de la batture de l'Isle-aux-Grues

6. Aubert Hamel, «La récupération et la mise en valeur des alluvions maritimes du SaintLaurent», L'agriculture, 20,3 (1963): 77-83; Jacques Rousseau, "La batture de l'île aux Grues et son importance économique", Société de Québec pour la protection des plantes, 1932-1934 (1934): 82-86; Robert-Lionel Séguin, «La récolte du jonc de cajeu et de la rouche à l'Île d'Orléans et à l'Île aux Grues ", Revue d'ethnologie du Québec, 2 (1975) : 9-22.

7. John Teal et Mildred Teal, Life and Death of the Salt Marsh (New York, Ballantine Books, 1969); J. Gauthier et al., Les marécages intertidaux..., op. cit.; Matthew G. Hatvany, Marshlands : Four Centuries of Environmental Change on the Shores of the St. Lawrence (Sainte-Foy, Les Presses de l'Université Laval, 2003). U. Larouche, «Les milieux humides... », loc. cit., 27; Stéphanie Harnois, "Entre terre et mer" : utilisations et perceptions environnementales des marais intertidaux de la Côte de Beaupré, mémoire de maîtrise (géographie), Université Laval, 2005 ; Catherine Plante, "Des marais et des hommes": nature et culture à l'Isle-aux-Grues, de l'époque amérindienne à aujourd'hui, mémoire de maîtrise (géographie), Université Laval, 2005. 
est particulier, les résidants de l'île ayant exploité les ressources de ce milieu sans avoir causé de dommages irréversibles.

\section{L'APPROCHE GÉOHISTORIQUE}

L'une des meilleures façons de comprendre et d'expliquer les changements environnementaux reste spatio-temporelle et conjugue la géographie et l'histoire ${ }^{8}$. Cette approche a été utilisée, notamment, dans l'étude des milieux humides du littoral européen, où l'on a aménagé, dès le XvII siècle, de nombreux polders ainsi que de grands ouvrages d'endiguement, afin de répondre aux besoins agricoles et urbains d'une population grandissante ${ }^{9}$. Les marais de la côte est du Canada, endigués à grande échelle par les Acadiens, ont également été étudiés. Wynn, Cormier, Hatvany, Butzer, Bleakney et Summerby-Murray en ont dressé un historique et ont mesuré l'impact de leur exploitation ${ }^{10}$. Sebold a fait de même pour certains marais de la Nouvelle-Angleterre et du New Jersey, ainsi que Park pour les marais et marécages de la Nouvelle-Zélande ${ }^{11}$. On retrouve éga-

8. Neil Roberts et Robin A. Butlin, «Ecological Relations in Historical Times: An Introduction», dans Neil Roberts et Robin A. Butlin, dir., Ecological Relations in Historical Times: Human Impact and Adaptation (Oxford \& Cambridge, Massachusetts, Blackwell, 1994), 1-14.

9. Henry Clifford Darby, The Medieval Fenland (Cambridge, Cambridge University Press, 1940); Lydie Goeldner, «L'évolution des endiguements littoraux: l'exemple de la côte allemande de la mer du Nord de 1954 à 1994 ", dans Emmanuel-Paul Désiré et Raymond Regrain, Sociétés humaines et milieux humides en Picardie (Paris, Comité des travaux historiques et scientifiques, 1996), 19-30; Nacima Baron-Yellès, Recréer la nature : écologie, paysage et société au marais d'Orx (Paris, Éditions Rue d'Ulm, 2000); Lydie Goeldner-Gianella, L'Allemagne et ses polders: conquête et renaissance des marais maritimes (Paris, Comité des travaux historiques et scientifiques, 2000); Nacima Baron-Yellès et Lydie Goeldner-Gianella, Les marais maritimes d'Europe atlantique (Paris, Presses universitaires de France, 2001), 32 ; Nacima Baron-Yellès, «Essai de géographie historique des paysages de marais de la Ria Formosa (Algarve, Portugal)", Actes du colloque "Aux rives de l'incertain: histoire et représentation des marais occidentaux du Moyen Âge à nos jours" (Poitiers, 2002).

10. Graeme Wynn, «Late Eighteenth-Century Agriculture on the Bay of Fundy Marshlands», Acadiensis, VIII,2 (1979): 80-89; Yves Cormier, Les aboiteaux en Acadie, hier et aujourd'hui (Moncton, Chaire d'études acadiennes, 1990); Matthew G. Hatvany, «The Origins of the Acadian Aboiteau: An Environmental Historical Geography of the Northeast ", Journal of Historical Geography, 30 (2002): 121-137; Matthew G. Hatvany, "Wedded to the Marshes": Salt Marshes and SocioEconomic Differenciation in Early Prince Edward Island ", Acadiensis, XXX,2 (2001): 40-55 ; Karl W. Butzer, «French Wetland Agriculture in Atlantic Canada and Its European Roots: Different Avenues to Historical Diffusion ", Annals of the Association of American Geographers, 92,3 (2002): 451-470; Sherman Bleakney, Sods, Soils and Spades: The Acadians at Grand Pré and Their Dykeland Legacy (Montréal, McGill-Queen’s University Press, 2004); Robert Summerby-Murray, «Interpreting Cultural Landscapes: A Historical Geography of Human Settlement on the Tantramar Marshes, New Brunswick» dans C. Stadel, dir., Themes and Issues of Canadian Geography III. Salzburger Geographische Arbeiten, (Salzburg, University of Salzburg), 34: 157-174.

11. Kimberly R. Sebold, "Low Green Prairies of The Sea : Economic Usage and Cultural Construction of The Gulf of Maine Salt Marshes, thèse de doctorat (histoire), University of Maine, 1998; 
lement la même approche dans les travaux de Hatvany à Kamouraska, de Harnois sur la Côte-de-Beaupré, de Plante à l'Isle-aux-Grues et de Carrier à l'île Verte et L'Isle-Verte. Tous ont documenté la relation temporelle entre les activités humaines et les environnements humides, démontrant ainsi l'importance agricole et écologique de longue date des marais du Saint-Laurent ${ }^{12}$.

Étudier la géographie historique environnementale d'un lieu - en l'occurrence l'Isle-aux-Grues et ses marais - implique d'utiliser une méthodologie particulière afin de documenter le rapport humain avec l'environnement, et ce, de manière diachronique, c'est-à-dire depuis les derniers quatre cents ans. Comme l'a rappelé Courville, tout événement a lieu dans un espace et tout espace évolue dans le temps; la géographie et l'histoire sont donc nécessairement complémentaires. Ainsi, entreprendre la géographie historique d'un lieu consiste à retracer les changements de ce lieu à travers le temps, en exploitant des sources qui renseignent sur les événements liés au territoire, à différentes échelles. Tandis que le géographe teste ses hypothèses et valide ses données à partir d'observations concrètes faites sur le terrain, l'historien le fait à partir de documents d'archives. Le géographe historien a recours à ces deux moyens ${ }^{13}$. En ajoutant une dimension environnementale à la démarche de la géographie historique, il fait de l'espace une source de première main, à partir de laquelle il documente l'impact humain sur cet espace.

Puisque certaines des données de base utiles à l'étude des marais sont à caractère socio-économique, il convenait d'établir un cadre temporel d'observations fondées sur ces caractéristiques. Le modèle développé par Courville pour expliquer la diversité du paysage québécois - succession de grands contextes de civilisation qui laissent l'impression d'une organisation en strates du territoire, qui tantôt composent, tantôt restent étrangères l'une à l'autre - pouvant ici être utile, il a été appliqué à l'histoire environnementale de l'Isle-aux-Grues et adapté pour tenir compte des

Kimberly R. Sebold, From Marsh To Farm: The Landscape Transformation of Coastal New Jersey (Washington, D.C., National Park Service, 1993); Geoff Park, Ngã Uruora-The Groves of Life : Ecology and History in a New Zealand Landscape (Wellington, Victoria University Press, 1995).

12. M. G. Hatvany, Marshlands..., op. cit.; Stéphanie Harnois, "Entre terre et mer»..., op. cit.; Catherine Plante, "Des marais et des hommes»..., op. cit.; Mathieu Carrier, La compréhension de la gestion historique des marais de l'Île Verte et de L'Isle-Verte sur leur développement actuel, mémoire de baccalauréat (géographie), Université Laval, 2006.

13. Serge Courville, Introduction à la géographie historique (Sainte-Foy, Les Presses de l'Université Laval, 1995), 5-6, 52. 
événements particuliers de cette histoire ${ }^{14}$. Ainsi, pour l'Isle-aux-Grues, le contexte amérindien débute longtemps avant la colonisation européenne et se termine vers 1646, date de la concession de la seigneurie de la Rivière-du-Sud, qui comprenait alors l'Isle-aux-Grues et l'Isle-aux-Oies. À l'époque, les Amérindiens pratiquaient la chasse, la pêche et la cueillette de plantes et de fruits dans les marais ${ }^{15}$.

L'arrivée des Européens marque le début d'un nouveau contexte agraire (ou colonial). De 1646 à environ 1890, l'exploitation des marais pour le foin de mer évolue et culminera dans la seconde moitié du XIx ${ }^{\mathrm{e}}$ siècle. Au cours de cette période, la population, qui atteint son maximum à la fin du siècle, pratique une économie agricole mixte, orientée vers la subsistance du ménage et la vente de surplus quand il y en a. Bien qu'il soit plutôt pauvre sur le plan des techniques, ce type d'agriculture permet de nourrir une population d'environ 600 habitants. Les marais sont un lieu d'appoint: en plus d'être des lieux de chasse et de pêche, ils servent de pâturages pour les animaux de l'île. On en récolte également le foin de mer.

Une période plus industrielle débute vers 1890 et se prolonge jusqu'en 1960-1980. L'économie de marché se développe, l'agriculture devient intensive et spécialisée autour de l'industrie laitière; la technologie s'améliore. Néanmoins, l'usage des marais reste le même que durant les xviI ${ }^{\mathrm{e}}$, $\mathrm{XVIII}^{\mathrm{e}}$ et $\mathrm{XIX}^{\mathrm{e}}$ siècles. Même la récolte du foin de mer perdure.

Enfin, quand s'amorce l'ère postindustrielle, dans les années 1980, l'industrie laitière domine et le tourisme de nature se répand. L'Isle-auxGrues s'étant dépeuplée, la disponibilité des ressources est devenue plus importante, ce qui aurait dû normalement favoriser l'agriculture. L'usage des marais, cependant, a évolué: on fauche moins le foin de mer - sauf pour «l'entretien» du haut marais - et seulement pour la production limitée d'un produit du terroir (le fromage). Ce qui l'emporte, cette fois, ce sont les loisirs de plein air: chasse, ornithologie, marche et écotourisme. Ces activités sont en effervescence et sont une véritable manne pour les insulaires, qui accueillent un flot continu de visiteurs durant l'été et l'automne (tableau 1).

14. Ibid., 75 .

15. J. Teal et M. Teal, Life and Death of..., op. cit., 19; Lorraine Guay, Le Saint-Laurent insulaire: étude géographique, thèse de doctorat (géographie), Université Laval, 2000, 332. 


\section{Tableau I}

Contextes de civilisation de l'Isle-aux-Grues et usages des marais

\begin{tabular}{|c|c|c|c|}
\hline $\begin{array}{c}\text { Contexte } \\
\text { de civilisation }\end{array}$ & Dates & $\begin{array}{l}\text { Utilisation } \\
\text { des marais }\end{array}$ & $\begin{array}{c}\text { Caractéristiques } \\
\text { socio-économiques }\end{array}$ \\
\hline Postindustriel & $1980-2005$ & $\begin{array}{l}\text { Chasse } \\
\text { Loisirs de plein air } \\
\text { Récolte du foin } \\
\text { Pêche }\end{array}$ & $\begin{array}{l}\text { Économie de marché } \\
\text { Agriculture intensive mono- } \\
\text { production } \\
\text { Ratio population-ressources } \\
\text { favorable } \\
\text { Forte mécanisation }\end{array}$ \\
\hline Industriel & $1890-1980$ & $\begin{array}{l}\text { Récolte du foin } \\
\text { Pâturage } \\
\text { Chasse } \\
\text { Pêche }\end{array}$ & $\begin{array}{l}\text { Économie de marché } \\
\text { Agriculture intensive mixte } \\
\text { Ratio population-ressources } \\
\text { défavorable vers favorable } \\
\text { Mécanisation simple des } \\
\text { techniques }\end{array}$ \\
\hline Colonial & $1646-1890$ & $\begin{array}{l}\text { Récolte du foin } \\
\text { Pâturage } \\
\text { Chasse } \\
\text { Pêche }\end{array}$ & $\begin{array}{l}\text { Économie de subsistance } \\
\text { Agriculture extensive mixte } \\
\text { Ratio population-ressources } \\
\text { favorable vers défavorable } \\
\text { Faible apport technologique }\end{array}$ \\
\hline Amérindien & Avant 1646 & $\begin{array}{l}\text { Chasse } \\
\text { Pêche } \\
\text { Cueillette }\end{array}$ & $\begin{array}{l}\text { Société nomade } \\
\text { Chasseurs cueilleurs } \\
\text { Ratio population-ressources } \\
\text { favorable }\end{array}$ \\
\hline
\end{tabular}

Source: Plante, 2005, 16.

\section{LE CONTEXTE DE CIVILISATION AMÉRINDIEN (JUSQU'EN I646)}

Rares sont les documents historiques qui font état de la relation entre les Amérindiens et les marais. La plupart des informations disponibles proviennent des fouilles archéologiques. En les comparant à la carte du poste de Québec dressée par Champlain en 1608 (Québec et ses environs en 1608), on constate que les Amérindiens vivaient en symbiose avec les milieux humides. C'est du moins ce qu'indique l'illustration de l'estran intertidal de la Côtede-Beaupré, où les Amérindiens pratiquaient la chasse et la pêche ${ }^{16}$.

16. Claude Chapdelaine et Pierre Lasalle, "Physical Environments and Cultural Systems in the Saint Lawrence Valley, 8,000 to 3,000 B. P.: A Multidisciplinary Framework", dans E. A. Bettis III, dir., Archaeological Geology of the Archaic Period in North America (Boulder, Colorado, Geological Society of America, Special Paper 297, 1995); Claude Chapdelaine, «La transhumance et les Iroquoiens du Saint-Laurent», Recherches amérindiennes au Québec, XXIII,4 (1993): 23-38; Claude Chapdelaine, "The Maritime Adaptation of the Saint Lawrence Iroquoians ", Man in the Northeast, 45 (1993): 3-19; Marcel Moussette, Île aux Oies. Interventions archéologiques de 1987 (Québec, CÉLAT, Université Laval, 1988). La carte de Champlain est reproduite dans Marcel Trudel, Atlas de la Nouvelle-France (Sainte-Foy, Les Presses de l'Université Laval, 1968), 190. 
Les premiers peuples autochtones qui ont fréquenté les îles de l'estuaire moyen du Saint-Laurent sont les Paléo-Amérindiens. Les vestiges laissés par ces peuples sur la Côte-du-Sud datent de 7000 à 3000 ans avant nos jours. De petites bandes de nomades ont visité l'Isle-aux-Grues et l'Isleaux-Oies et y ont établi des campements saisonniers afin d'en exploiter les ressources ${ }^{17}$. Fruits, racines, plantes médicinales, mais surtout sauvagine et poissons (dont l'anguille), tout était mis à contribution, permettant même des réserves pour le retour aux campements d'hiver ${ }^{18}$. Cet attrait pour les marais et les estrans ne fait d'ailleurs aucun doute dans les récits du jésuite Paul Le Jeune. Rédigés avant que la seigneurie de la Rivière-duSud ne soit concédée, à l'occasion d'un voyage dans l'estuaire en compagnie d'un groupe d'Amérindiens, il donne la description suivante de l'Isle-aux-Grues et de l'Isle-aux-Oies:

Le lendemain nous quittames cette Isle [Orléans] pour entrer dans une autre appellée Ca chibariouachcate, nous la pourrions nommer l'Isle aux Oyes blanches, car j'y en vis plus de mille en une bande [...] Elle est entre-taillée de baies pleines de vases, il s'y retire si grande quantité de gibier [les oies des neiges] et de plusieurs espèces que je n'ai point vu en France, qu'il le faut quasi voir pour le croire ${ }^{19}$.

Pour l'Amérindien, le lieu est donc important et fait partie de son aire d'approvisionnement. Cependant, son exploitation semble saisonnière et sert surtout à accumuler des réserves pour le retour en forêt l'hiver.

\section{LE CONTEXTE DE CIVILISATION AGRAIRE (1646- 1890)}

La période qui suit amène un autre type d'exploitation des marais. À la chasse et à la pêche s'ajoute cette fois une activité plus sédentaire : l'agriculture. Les premiers colons s'y adonnent sans technologie très avancée, mais en récoltant tout de même des quantités impressionnantes de foin de mer. C'est au cours de cette période que se développent une socioéconomie et des rapports aux marais très étroits, comme le révèle la documentation disponible.

17. Alain Laberge, dir., Histoire de la Côte-du-Sud (Québec, Institut québécois de recherche sur la culture, 1993), 58; Charles A. Martijn, «The Iroquoian Presence in the Estuary and Gulf of the Saint Lawrence River Valley: A Reevaluation", Man in the Northeast, 40 (1990): 58; J. Teal et M. Teal, Life and Death of..., op. cit., 19; L. Guay, Le Saint-Laurent insulaire..., op. cit., 332.

18. A. Laberge, Histoire de la Côte-du-Sud..., op. cit., 56; William Cronon, Changes in the Land (New York, Hill and Wang Editions, 1983), 54; C. Chapdelaine, «La transhumance»..., op. cit.

19. Reuben Gold Thwaites, dir., The Jesuit Relations and Allied Documents: Travels and Explorations of the Jesuit Missionaries in New France 1610-1791 (New York, Pageant Book Company, 1959), 78. 
Au début de la colonisation européenne de la vallée du Saint-Laurent, la plupart des terres étaient recouvertes de forêts. Seuls les campements amérindiens, les plans d'eau, les tourbières et les prairies naturelles, souvent associées aux marais, étaient dégagés. Riches en foin de mer, ces prairies étaient très recherchées par les colons, qui voyaient là un moyen de nourrir à peu de frais ou d'efforts leur bétail. L'un des plus anciens documents faisant état de cette valorisation provient des religieuses de l'Hôtel-Dieu de Québec, propriétaires de l'Isle-aux-Oies de 1713 à 1964. Ayant besoin de foin pour nourrir leur bétail, elles achètent l'île et une partie du marais qui la sépare de l'Isle-aux-Grues. Quelque temps plus tard, en 1751, elles visitent l'île et la décrivent ainsi :

Enfin quand nous fument au débarquement, nous découvrîmes des batures [sic] fort étendues, et des grandes prairies, que la marée haute nous avait cachées $[\ldots]$ Nous vîmes revenir un beau troupeau de vaches; l'abondance des pâturages, fait qu'elles y sont toujours grasses, et que leur lait est excellent et le beurre meilleur que par tout ailleurs ${ }^{20}$.

Outre les documents écrits, d'autres sources permettent d'apprécier la valeur des marais. C'est le cas de certaines cartes de l'époque coloniale, qui montrent bien l'importance socio-économique et environnementale du haut marais de l'Isle-aux-Grues, et ce, malgré sa faible superficie comparée à la région cartographiée. La carte la plus révélatrice à cet égard est celle de Deshayes de 1715 (figure 3). L'auteur a recours à une trame représentant des touffes d'herbe pour délimiter le haut marais de l'Isle-aux-Grues. L'effet est saisissant: en dépit d'une occupation humaine de presque cent ans, la carte montre un milieu en apparence totalement naturel, sans transformation anthropique. Un autre document est tout aussi révélateur: c'est la carte de Murray de 1761, Plan of Canada or the Province of Quebec from the uppermost settlements to the Island of Coudre. En plus de montrer la grande superficie du haut marais, l'ouvrage reproduit en détail le réseau hydrographique qui drainait alors le marais. L'arpenteur général du Bas-Canada, Joseph Bouchette, ne sera pas en reste. Dans son ouvrage sur la topographie du Bas-Canada (1815), il note à son tour la grande abondance de foin dans les marais de l'Isle-aux-Grues qui, selon lui, était suffisant pour nourrir un

20. M. G. Hatvany, Marshlands..., op. cit.; Jean-Marie Lemieux, L'Île-aux-Grues et l'Ille-aux-Oies: les îles, les seigneurs, les habitants, les sites et monuments historiques (Montréal, Leméac, 1973, 1978); Mère A. M. J. Saint-Ignace, Histoire de l'Hôtel-Dieu de Québec (Montauban/Paris, Jérosme Légier/ Claude-Jean-Baptiste Herissant, libraire, 1751), 516. 
troupeau de 3000 têtes $^{21}$. Et, comme ses prédécesseurs, il n’indique pas de changement environnemental important.

\section{Figure 3}

De la Grande Rivière de Canada appellée par les Européens de St. Laurens

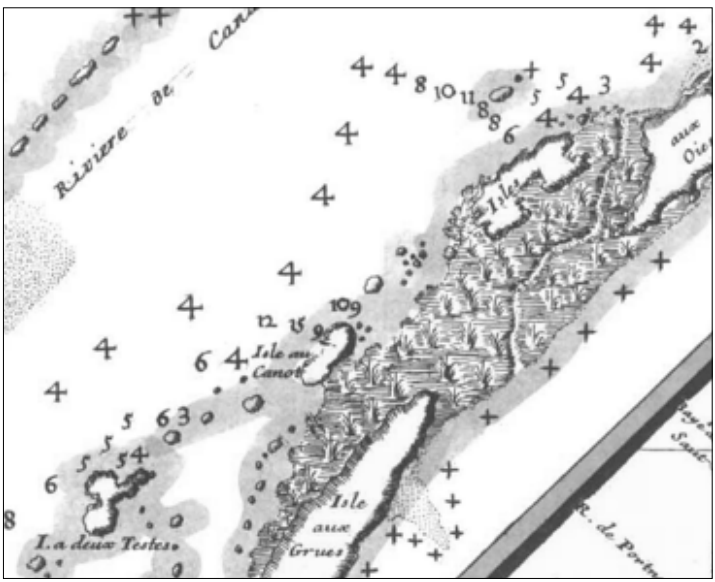

Source: Deshayes, 1715.

Les documents rédigés par les notaires sont une autre source très précieuse pour étudier la perception et l'utilisation des marais par les habitants de l'île. Avant que les censitaires deviennent propriétaires et exploitants de lots de batture au tout début du Xix ${ }^{\mathrm{e}}$ siècle, ils devaient, par obligation, payer au seigneur le foin qui y était récolté. Or, à la fin du xviII siècle, le seigneur de l'île demande des arrérages de plus de 9000 livres à ses fermiers pour dettes de foin. Ces chiffres donnent une idée de la valeur économique du foin de mer à cette époque ${ }^{22}$. Par la suite, l'exploitation des marais sera régie

21. Jean Deshayes, De la Grande Rivière de Canada appellée par les Européens de St. Laurens. Document cartographique, levé et dressé par Deshayes, 1 carte en 2 feuilles (Paris, Chez N. de Fer, 1715, fac-similé de l'édition originale de 1706), Bibliothèque et Archives nationales du Québec, banque de cartes et plans en ligne : http://bibnum2.banq.qc.ca/bna/cargeo/accueil.htm cote : G 1116 S1A8 1980 CAR ; James Murray, Plan of Canada or the Province of Quebec from the Uppermost Settlements to the Island of Coudre. Document cartographique. 1 carte en 76 coupures (Ottawa, Archives publiques du Canada, collection nationale de cartes et plans, 1762); Joseph Bouchette, Description topographique de la province du Bas-Canada avec des remarques sur le Haut-Canada et sur les relations des deux provinces avec les États-Unis de l'Amérique (Londres, T. Davison, 1815); Joseph Bouchette, This Topographical Map of the Districts of Quebec, Three Rivers, St. Francis and Gaspé, Lower Canada. Document cartographique, gravure de J. et C. Walker. 1 carte sur 6 feuillets, échelle $1: 253,440$ (London, James Wyld, geographer to the King, Charing Cross, May $2^{\text {nd }}$., 1831).

22. Les obligations n'étaient pas des baux devant notaire, mais des ententes verbales. Jules Vézina, La Côte-du-Sud: histoire et généalogie d'un archipel (Isle-aux-Grues, Québec, Association historique, 1994), 67; Extraits du greffe du notaire Jean-Baptiste Faribault, 1797, tiré du fonds d’archives privé de Jules Vézina. 
par des baux signés devant notaire. Elle donnera une plus-value aux lots situés sur le bord du fleuve. Ainsi, calculée pour la période 1874-1898 (tableau 2), à partir de sources notariales, la valeur moyenne à l'arpent carré d'un lot de marais est de deux à cinq fois plus élevée que celle d'un lot régulier en raison de la présence du foin de mer, qui ne coûte rien à produire et qui autorise des gains substantiels sur le marché.

Tableau 2

Valeur marchande des lots de marais et des lots de terre agricole, Isle-aux-Grues, fin du XIXe siècle

\begin{tabular}{|c|c|c|c|}
\hline \multicolumn{2}{|c|}{ Haut marais } & \multicolumn{2}{|c|}{ Terre (île) } \\
\hline Date de vente & $\begin{array}{c}\text { Coût par arpent carré } \\
\text { (en } \$ \text { ) }\end{array}$ & Date de vente & $\begin{array}{c}\text { Coût par arpent carré } \\
(\text { en } \$)\end{array}$ \\
\hline 1874 & 76,00 & 1842 & 23,52 \\
\hline 1874 & 101,20 & 1875 & 48,81 \\
\hline 1874 & 27,78 & 1876 & 29,80 \\
\hline 1874 & 45,72 & 1876 & 18,75 \\
\hline 1887 & 22,78 & 1887 & 13 (avec bâtiments) \\
\hline 1894 & 28,03 & 1895 & 0,75 \\
\hline 1894 & 49,50 & 1896 & 4,50 \\
\hline 1898 & 48,00 & & \\
\hline Moyenne & 49,88 & Moyenne & 19,88 \\
\hline
\end{tabular}

Les valeurs en livres ont été converties selon le taux établi dans la deuxième moitié du XIX ${ }^{\mathrm{e}}$ siècle où une livre vaut $4 \$$ (ANQ, 1858).

Source: Actes notariés de F.X. Talbot (1842), L.-A. Beaubien (1874), F.X. Gendreau (1874, 1875, 1876), H. Hébert (1887), N. Gauthier (1894, 1895, 1896, 1898), fonds d’archives privé de Jules Vézina.

Des accords (baux) de location de lots de marais entre le seigneur et les cultivateurs de l'Isle-aux-Grues au début du XIX ${ }^{\mathrm{e}}$ siècle donnent, d'ailleurs, d'intéressants renseignements sur la façon de récolter le foin ${ }^{23}$. Selon l'un de ces baux daté de 1817 , les cultivateurs ont alors le droit de «faire [...] sur la batture de ladite Isle aux Grues [...] tous les foins et fourrages qu'ils [...] pourront avoir besoin chaque année durant le susdit temps pour la nourriture de leurs bestiaux». Mais, en revanche, chaque cultivateur doit livrer au seigneur «le tiers en qualité et quantité de tous les foins et fourrages

23. Daniel McPherson a acquis la seigneurie en 1802. Il a alors consenti à vendre certains lots de batture. Des baux de location de lots de batture ont aussi été accordés aux cultivateurs qui ne pouvaient se permettre de les acheter. J.-M. Lemieux, L'T̂le-aux-Grues et..., op. cit., 95. 
généralement quelconques qu'ils pourront faire». De plus, les foins récoltés sur la batture doivent être "dûment conditionnés et mis en mûles et bien couvertes et dûment enfaitées et liées et élevées convenablement sur des échafauds afin que la mer ne puisse leur faire aucun dommage». Le seigneur exige aussi que les cultivateurs prennent soin des marais et qu'ils fassent «brûler le vieux foin s'il en reste, tous les ans» afin de favoriser la croissance des plantes qui seront récoltées au printemps suivant. Ils devront éliminer le "rapport» (les débris marins poussés sur le haut marais par les grandes marées) et payer au bailleur "la somme de deux livres dix chelins [shillings] courant, par chaque arpent de bature de front, sur toute la profondeur de ladite bature de l'Isle aux Grues ${ }^{24}$.»

C. H. Farnham, un touriste américain qui a visité l'île, donne également une description très vivante des méthodes de récolte du foin de mer dans les pages du Harper's New Monthly Magazine de 1888. Selon lui, «salt-meadows connect the Isle aux Grues with its twin the Isle aux Oies; at low tide cattle were feeding on rushes about the schooners $[\ldots]$ These meadows are celebrated shooting-grounds [la chasse aux oies]». Il poursuit: «if you happen there near the full-moon of September, you will find the flats [marais] alive with mowers cutting rushes [foin de mer]». Il termine son récit en mentionnant que «the cutting of rushes for hay at St. Thomas [Montmagny], L'Islette, Beaupré, and other places [...] is a characteristic scene $[\ldots]$ one of the prettiest scenes in Canada ${ }^{25} »$. Cette tradition et celle du remisage du foin en meules sur des "échafauds» (structures de bois) persisteront jusqu'au milieu du $\mathrm{xx}^{\mathrm{e}}$ siècle (figure 4$)^{26}$. Les récits de voyage et les baux témoignent de l'importance accordée à la qualité des récoltes et à la gestion du marais pour que tous les insulaires puissent $\mathrm{y}$ avoir accès. Cet avantage ne se reflète pas seulement dans les récits et dans les baux, mais également dans les données des recensements des $\mathrm{XIx}^{\mathrm{e}}$ et $\mathrm{xx}^{\mathrm{e}}$ siècles.

Le privilège que constitue l'accès aux marais a en effet été mesuré par une étude comparative de deux séries de recensements: l'une pour la période de 1851 à 1871 (listes nominatives), soit en pleine période agraire ${ }^{27}$,

24. Extraits du greffe du notaire Abraham Larue, 1817, tiré du fonds d’archives privé de Jules Vézina.

25. C. H. Farnham, «The lower St. Lawrence», Harper's New Monthly Magazine, 77,462 (1888): 820-824.

26. Entrevues à l'Isle-aux-Grues avec Simon Painchaud (2002), Bertrand Vézina (2002) et Julien Vézina (2002), enregistrements et notes en possession de Catherine Plante.

27. Paroisses de Saint-Antoine-de-l'Isle-aux-Grues (Isle-aux-Grues et Isle-aux-Oies seulement), de l'île Verte, de Saint-Ferréol-les-Neiges, de Saint-François-de-la-Rivière-du-Sud, Canada, Recensement du Canada Est (Archives nationales du Québec, Québec, 1851, 1861, 1871). 
Figure 4

Hommes enfaîtant une meule de foin sur un échafaud

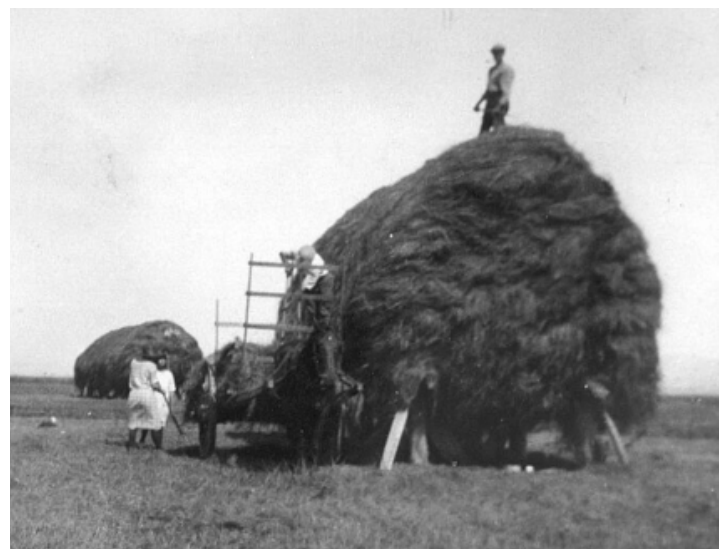

Source: Fonds Jules Vézina, 1715.

et l'autre pour la période de 1881 à 1956 (copies agrégées) ${ }^{28}$, en contexte industriel. La comparaison sur les plans de la population et du nombre de fermes a été faite entre quatre sites semblables, tous situés à l'est de la ville de Québec. Ce sont l'Isle-aux-Grues (et l'Isle-aux-Oies), l'île Verte (120 km à l'est), Saint-François-de-la-Rivière-du-Sud, un terroir plus ancien dans la région de Montmagny, et Saint-Ferréol-les-Neiges, sur la rive nord de l'estuaire, presque en face de l'Isle-aux-Grues. Cette dernière paroisse était, en 1851, récemment établie, donc encore en défrichement et sans accès aux marais ${ }^{29}$.

Les variables du recensement les plus intéressantes pour cette étude concernent les quantités de foin récolté, le nombre d'animaux possédés et la quantité de beurre produit. Les questions ayant changé d'un recensement à l'autre, il n'a pas toujours été possible de comparer certaines variables dans le temps. Cependant, les données disponibles ont quand même permis certains constats. Ainsi, la figure 5 indique que le nombre de bottes de foin récolté de 1851 à 1871 est en moyenne deux fois plus

28. Paroisses de Saint-Antoine-de-l'Isle-aux-Grues (Isle-aux-Grues et Isle-aux-Oies seulement), de Notre-Dame-des-Sept-Douleurs, de Saint-Ferréol-les-Neiges, de Saint-François-de-la-Rivièredu-Sud, Canada, Recensement du Canada, 1881, 1891, 1911, 1931, 1941, 1951, 1956.

29. Notez que dans la section d'analyse couvrant les années 1851 à 1871 , seulement les fermes de cinq arpents ou plus ont été prises en compte. Sur la méthodologie de comparaison des recensements entre différentes sous-divisions, voir C. Plante, "Des marais et des hommes"..., op. cit., 99-157; Régis Thibeault, L'essor de l'industrie laitière et du capitalisme agraire au Saguenay, XIX ${ }^{e}$ et $x x^{e}$ siècles, thèse de doctorat (histoire), Université Laval, 2002. 
élevé à l'Isle-aux-Grues qu'à Saint-François-de-la-Rivière-du-Sud, alors que les quantités récoltées dans les deux autres paroisses sont minimes. Également, on constate que le nombre d'animaux possédés par ferme passe de 7 à 26 à l'île Verte entre 1851 et 1871 , de 17 à 22 à Saint-Françoisde-la-Rivière-du-Sud et de 41 à 33 à l'Isle-aux-Grues, Saint-Ferréol-lesNeiges n'enregistrant pas de changement important.

Figure 5

Nombre moyen de bottes de foin récolté par ferme de 5 arpents ou plus, I85I

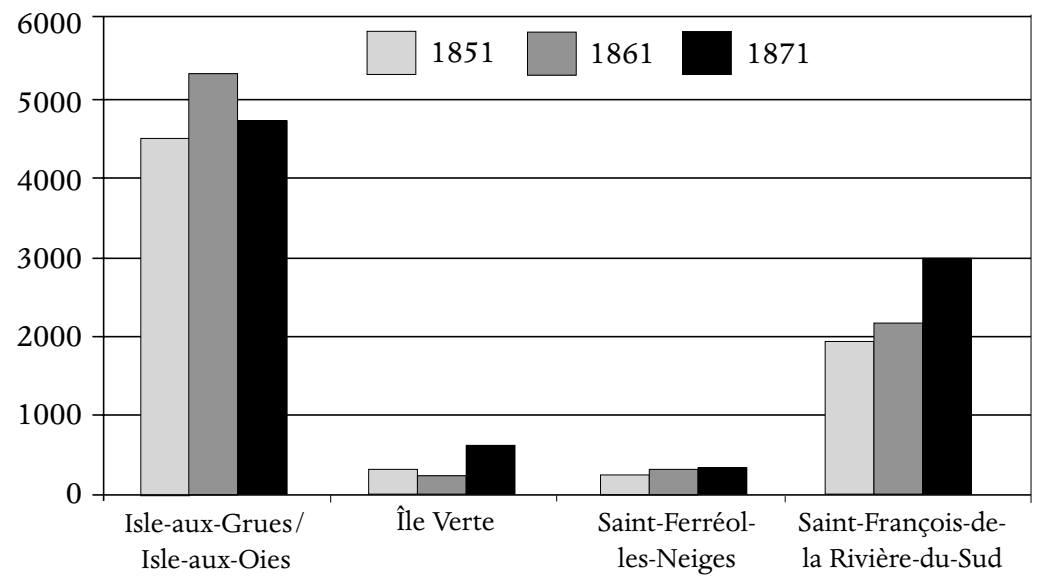

Ces données appellent quatre commentaires. D’abord, même si le nombre de bêtes diminue à l'Isle-aux-Grues, l'accès aux ressources naturelles du marais reste important. Comment, sinon, aurait-on pu nourrir un tel troupeau? Ensuite, avec plus de deux tonnes de foin disponibles par tête de bétail, l'Isle-aux-Grues est loin de manquer de fourrages (tableau 3). Il en va autrement pour l'île Verte et surtout Saint-Ferréol-les-Neiges, où la ferme dispose de moins d'une tonne de foin par animal, une situation qui peut entraîner une perte de bétail l'hiver. De plus, ces données montrent que les grandes quantités de foin de mer récolté à l'Isle-aux-Grues ont permis de vendre les surplus sur la Côte-du-Sud et sur la rive nord, comme le recensement de 1851 le note: "Chaque habitant y possède sur la batture ordinairement 40 à 50 arpents $[\ldots] \&$ en retire une très grande quantité de foin, dont une partie sert à la nourriture de ses animaux $\&$ l’autre partie $[\ldots]$ à la vente ${ }^{30}$.» Enfin, ces données révèlent que le nombre

30. Recensement du Canada Est, 1851-1852, comté de l’Islet, folio 24, 23. 
de vaches laitières possédées a permis une grande production de beurre. Avec plus de cinq vaches laitières par ferme en 1851, l'Isle-aux-Grues se démarque de Saint-François-de-la-Rivière-du-Sud, qui en possède en moyenne 3,5 (figure 6). L'écart avec l'île Verte et Saint-Ferréol-les-Neiges est d'au moins trois vaches de plus pour chaque fermier de l'Isle-auxGrues. Quant au nombre de livres de beurre produit par ferme de 1851 à 1871 et en 1891, la domination de l'Isle-aux-Grues sur les trois autres lieux de la comparaison est marquée, en raison de la grande quantité de foin de mer disponible qui peut nourrir plus de vaches laitières.

Tableau 3

Nombre moyen de tonnes de foin disponibles par animal*, |85|-|87|

\begin{tabular}{cccc}
\hline $\begin{array}{c}\text { Date du } \\
\text { recensement }\end{array}$ & $\begin{array}{c}\text { Tonnes de } \\
\text { foin récoltées }\end{array}$ & $\begin{array}{c}\text { Bétail mangeur } \\
\text { de foin }^{\star}\end{array}$ & $\begin{array}{c}\text { Tonnes de foin } \\
\text { par animal }\end{array}$ \\
\hline
\end{tabular}

Isle-aux-Grues

$\begin{array}{llll}1851 & 1648,10 & 802 & 2,05 \\ 1861 & 2074,12 & 927 & 2,24 \\ 1871 & 1946,80 & 757 & 2,57\end{array}$

Île Verte

$\begin{array}{lrcr}1851 & 12,00 & 7 & 1,71 \\ 1861 & 52,12 & 168 & 0,31 \\ 1871 & 178,40 & 248 & 0,72\end{array}$

Saint-Ferréol-les-Neiges

$\begin{array}{llll}1851 & 205,68 & 485 & 0,42 \\ 1861 & 217,68 & 506 & 0,43 \\ 1871 & 408,14 & 998 & 0,41\end{array}$

Saint-François-de-la-Rivière-du-Sud

$\begin{array}{llll}1851 & 4143,60 & 2313 & 1,79 \\ 1861 & 2058,80 & 1445 & 1,42 \\ 1871 & 4121,42 & 1631 & 2,53\end{array}$

1 botte vaut 16 livres

1 tonne 2000 livres

${ }^{\star}$ Chevaux et autre bétail (excepté les moutons et les porcs)

Source: Recensements du Canada (agricoles), 1851, 1861, 1871 et 1891

Ce que montrent ces chiffres, en fait, est un rapport durable entre les habitants de l'Isle-aux-Grues et le milieu humide. Pour eux, le marais et ses ressources étaient au cœur de l'économie locale. Exploités pour assurer la survie du groupe et lui fournir ses surplus, ils furent à la fois 
Figure 6

Nombre moyen de vaches laitières par ferme de 5 arpents ou plus, |85I-|87|

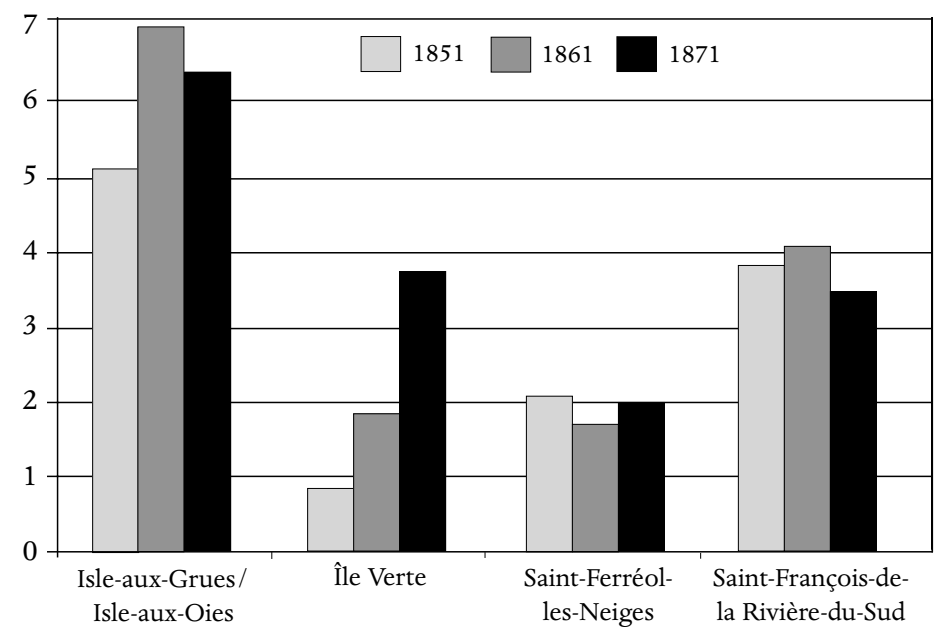

une source de revenus et un mode de vie. Cette symbiose avec le milieu naturel se maintiendra pendant plus de deux siècles, jusqu'à ce que la modernisation de l'agriculture sur la Côte-du-Sud ne vienne transformer ce rapport.

\section{LE CONTEXTE DE CIVILISATION INDUSTRIEL (1890- 1980)}

Comme partout sur la Côte-du-Sud, le contexte de civilisation industriel est synonyme, à l'Isle-aux-Grues, d'une spécialisation de l'agriculture autour de l'industrie laitière ${ }^{31}$. Les données agricoles pour la période 18811956 montrent que l'importance du foin de mer s'est maintenue jusqu'au milieu du $\mathrm{xx}^{\mathrm{e}}$ siècle. Les quantités de foin récolté restent plus élevées à l'Isle-aux-Grues que pour les autres sites comparés, soit au moins deux fois supérieures. Peut-être est-ce en raison des canalisations (ditching) faites par les insulaires dans le haut marais, qui commencent très tôt dans la période. Les canaux, creusés pour la plupart entre les lots de batture, aident à l'écoulement de l'eau, donc à la réduction de l'humidité, et favorisent du même coup le transport du foin avec des chevaux, de même que la croissance des espèces de foin de mer les plus recherchées, soit les 
carex $^{32}$. De 1891 à 1956, le nombre moyen d'animaux par ferme augmente constamment à l'Isle-aux-Grues, signe de l'abondance de nourriture procurée par la disponibilité du foin de mer, même au $\mathrm{xx}^{\mathrm{e}}$ siècle. Quant au nombre moyen de vaches laitières par ferme, l'Isle-aux-Grues se compare encore avantageusement aux autres milieux. On y dénombre en effet au moins 10 vaches de plus par ferme qu'à l'île Verte et Saint-Ferréol-lesNeiges, et environ cinq de plus en moyenne qu'à Saint-François-de-laRivière-du-Sud (figure 7).

Figure 7

Nombre moyen de vaches laitières par ferme, $|89|-1956$

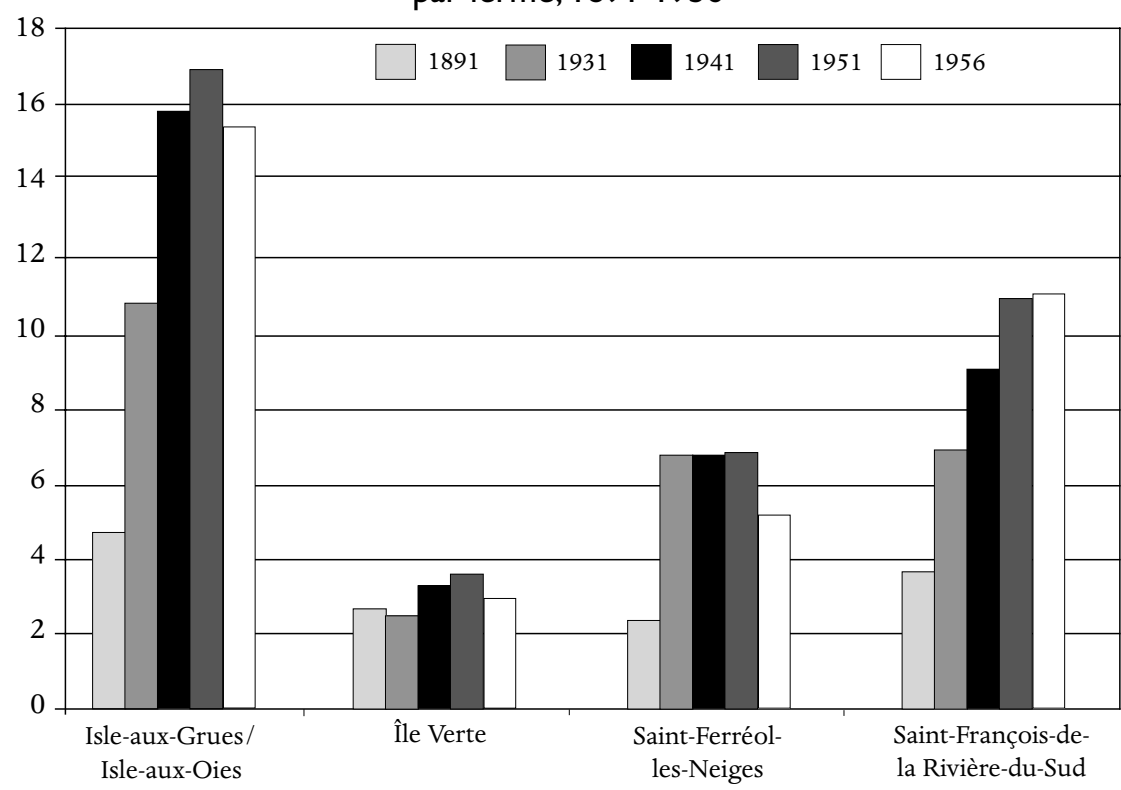

Les choses changent avec le début de l'agriculture moderne. Dès la seconde moitié du $\mathrm{xIX}^{\mathrm{e}}$ siècle, les agronomes commencent à se demander s'il est possible d'exploiter les marais plus intensivement. Plus tard, vers la fin du $\mathrm{XIX}^{\mathrm{e}}$ siècle, on entreprend des expériences scientifiques dans les collèges d'agriculture. Elles montrent que le foin de mer est de $15 \%$ à $20 \%$ moins nutritif que le foin cultivé. Le résultat ne se fait pas attendre:

32. Entrevue avec Bertrand Vézina, Isle-aux-Grues, 2003, enregistrement et notes en possession de Catherine Plante. Concernant la canalisation des marais, voir K. R. Sebold, Low Green Prairies, op. cit., et New Jersey Agricultural Experiment Station, The New Jersey Salt Marsh and Its Improvement, $\mathrm{n}^{\circ} 207$ (New Brunswick, New Jersey Agricultural Experiment Station, 1907), 13. 
bientôt, on assiste à la lente mise de côté du foin de mer au profit du foin cultivé $^{33}$. La décroissance de la population locale et régionale ainsi que le quasi-abandon de la culture de la pomme de terre feront le reste. Disposant désormais de plus de terres, la ferme produit ses fourrages, un tournant pris dès les années 1950 sur l'Isle-aux-Grues ${ }^{34}$.

Cette réorientation de l'agriculture locale n'a cependant pas totalement éliminé la récolte du foin de mer, laquelle jouait depuis les années 1950 le rôle de substitut en cas de manque de fourrage cultivé. Des photographies aériennes de 1964 montrent que le foin de mer est encore récolté et laissé sur le haut marais en meules sur des échafaudages. On fauche aussi pour "entretenir» le haut marais et éviter la croissance de plantes indésirables, pour le nettoyer des débris laissés par les grandes marées et pour faciliter la circulation au temps de la chasse, activité pratiquée depuis le début de la colonisation. Mais dans l'ensemble, la mise en valeur des marais connaît une lente décroissance, accompagnée d'une disparition des activités traditionnelles.

C'est ainsi que de grandes superficies de zones intertidales disparaissent, pour adapter les écosystèmes aux nouveaux besoins de la société et surtout à ses nouvelles idées en matière de développement. À Kamouraska, cette destruction se fait avec l'aide des agronomes. Sur la Côte-de-Beaupré, elle est le fait de la croissance urbaine ${ }^{35}$. Seules certaines localités, comme l'Isle-aux-Grues, résistent au changement. Là, il n'y a pas de transformation profonde des marais; les résidants de l'île continuent plutôt d'exploiter le milieu, surtout parce que les retombées du foin de mer sont importantes et, peut-être aussi, parce que les habitants sont insulaires, et donc moins sensibles aux influences extérieures. Cependant, on peut se demander jusqu'où ira cette fidélité, si l'on tient compte du coût des endiguements et du faible nombre d'agriculteurs disponibles pour l'assumer ${ }^{36}$.

33. M. G. Hatvany, Marshlands..., op. cit. 66-68; J. B. Lyndsay et B. K. Jones, «The Feeding Value of Salt-Marsh Hay ", Hatch Experiment Station of the Massachusetts Agricultural College, 52 (1898): $3-48$.

34. A. Laberge, Histoire de la Côte-du-Sud..., op. cit., 379-382, 451.

35. M. G. Hatvany, Marshlands..., op. cit., 73-124, 154-155 ; S. Harnois, "Entre terre et mer»..., op. cit.

36. Entrevues à l'Isle-aux-Grues avec Julien Vézina (2002), Bertrand Vézina (2002), Simon Painchaud (2002) et Gaston Bernier (2002), enregistrements et notes en possession de Catherine Plante. 


\section{LE CONTEXTE DE CIVILISATION POSTINDUSTRIEL (1980-)}

La dernière partie du $\mathrm{xx}^{\mathrm{e}}$ siècle a amené une technologie agricole plus développée, mais aussi un renouveau dans l'utilisation du haut marais de l'Isle-aux-Grues. Bien que moins exploité pour le foin de mer, le haut marais continue d'être un haut lieu de chasse à la sauvagine. Avec le passage chaque automne de plus de 100000 oies des neiges sur la batture, l'île et son haut marais se transforment en une grande pourvoirie. Cette exploitation du haut marais est gérée de façon soutenue et tous les emplacements de chasse sont soigneusement distribués. Il s'agit d'une activité qui raffermit les divisions cadastrales du marais, donc l'appropriation du territoire, et qui en consolide également la propriété, à la fois par des particuliers et par des pourvoiries ${ }^{37}$. Par ailleurs, une nouvelle activité de loisir, aussi liée à l'abondance de la faune aviaire sur le marais, est en plein essor à l'Isle-aux-Grues : le tourisme de plein air. Le haut marais regorge d'espèces fauniques rares et de plantes, dans un environnement dépaysant. Les ornithologues sont attirés par l'île et ses marais, car ceux-ci sont des milieux propices à la nidification de plusieurs espèces ${ }^{38}$.

Parallèlement à la chasse et au tourisme de nature, l'agriculture gruoise continue d'être prolifique grâce à l'industrie fromagère. Récemment, un nouveau fromage, fait à partir de lait de vache se nourrissant presque uniquement de foin de batture, a été commercialisé comme produit du terroir ${ }^{39}$. C'est dire à quel point l'histoire ici a su s'imposer. Aujourd'hui, le haut marais garde ses allures de milieu naturel, mais il conserve toutes ses symbioses.

\section{DES SIÈCLES D'EXPLOITATION DURABLE}

Les données qui précèdent montrent plusieurs siècles d'exploitation économique et culturelle des marais de l'Isle-aux-Grues, sans qu'il n'y ait eu pourtant de détérioration radicale de l'environnement. La paléoécologie confirme ce sentiment et permet de retracer l'évolution d'un environnement et de mesurer les changements qu'il a connus grâce à des sources proxy (c'est-à-dire à des données physiques, dans le cas où aucun document d'archive n'existe $)^{40}$.

37. Site Internet de la Corporation de la sauvagine de l'Isle-aux-Grues, http:/ / www.isle-auxgrues.com/activites/chasse.html

38. Ibid.

39. Rachel Brillant, «Des Suisses brunes à l'Île-aux-Grues», La semaine verte (Émission télévisée hebdomadaire, télévision de Radio-Canada), 27 février 2005.

40. Barry G. Warner, dir., Methods in Quaternary Ecology, reprint series 5 (Geoscience Canada, 1990), 53-63; Najat Bhiry et Louise Filion, "Analyse des macrorestes végétaux », dans Serge Payette 
Se fondant sur le prélèvement d'échantillons de sol et sur l'analyse macrofossile (identification de graines de plantes, de feuilles, de tiges ou de toute autre pièce végétale ou animale), Plante a récemment mené une étude paléoécologique afin de comparer l'évolution de deux marais, dont le haut marais de l'Isle-aux-Grues, qui est susceptible d'avoir été physiquement modifié par l'action humaine. L'autre est situé à la pointe nordest de l'Isle-aux-Oies et jouait le rôle de milieu témoin; selon les données disponibles, ce marais témoin a été peu utilisé aux fins agricoles. À l'aide des échantillons de sol de $100 \mathrm{~cm}$ de longueur (appelés carottes), les macrofossiles ont été identifiés, comptés et datés. Selon l'assemblage des espèces végétales retrouvées à un niveau donné dans les carottes, différentes phases de l'évolution du marais ont pu être retracées en examinant l'écologie de chaque plante ${ }^{41}$.

Selon l'étude de Plante, les données macrofossiles du haut marais de l'Isle-aux-Grues indiquent que, malgré la récolte annuelle de plusieurs centaines de tonnes de foin de mer chaque année pendant presque 400 ans, il n'y a pas eu de changement majeur de l'environnement. Par ailleurs, des variations dans l'humidité de l'écosystème ont été retracées. À titre d'exemple, la disparition des graines de quenouille, une plante exigeant des conditions saturées en eau, à $20 \mathrm{~cm}$ de la surface, révèle un assèchement du milieu. La date de cette disparition concorde avec la période où les insulaires de l'Isle-aux-Grues ont commencé à creuser des canaux de drainage dans le marais à l'aide de machineries vers la fin du XIX ${ }^{\mathrm{e}}$ siècle. L'abondance actuelle de salicaire pourpre et de roseau commun sur le haut marais pourrait être associée aussi aux activités humaines, surtout à la canalisation et à la diminution de la fauche du foin sur la batture, qui aurait permis la mise en place de telles espèces envahissantes (non indigènes) en provenance des rives de l'estuaire ${ }^{42}$. En comparant les données macrofossiles de la carotte témoin et de celle prélevée dans le haut marais de l'Isle-aux-Grues jadis exploité aux fins agricoles, il s'avère que l'impact anthropique n'est pas absent, mais plutôt subtil, et ce, malgré l'exploitation du marais pendant plusieurs siècles.

et Lyne Rochefort, dir., Écologie des tourbières du Québec-Labrador (Sainte-Foy, Les Presses de l'Université Laval, 2001), 259-273.

41. C. Plante, «Des marais et des hommes»..., op. cit., 165-183.

42. L. A. Meyerson et al., "A Comparison of Phragmites Australis in Freshwater and Brackish Environments in North America ", Wetlands Ecology and Management, 8 (2000): 89-103; Benoît Gauthier, Recherches floristiques et écologiques sur l'hydrolittoral de l'archipel de Montmagny, mémoire de maîtrise (agriculture), Université Laval, 1972, 116. 


\section{CONCLUSION}

Debout au milieu du haut marais de l'Isle-aux-Grues, on a l'impression d'être entouré d'une nature sauvage, d'un milieu où les êtres humains sont quasi absents. Cela est loin d'être le cas, puisque depuis plus de 400 ans, les résidants de l'île vivent étroitement avec les marais. Il semble que l'évolution des perceptions, de l'exploitation et éventuellement de l'aménagement du haut marais de l'Isle-aux-Grues (contrairement à certains marais de l'estuaire) soit liée au fait que les activités humaines y aient évolué parallèlement aux processus naturels de l'écosystème du marais. Ce qui frappe dans cette étude des milieux humides de l'Isle-aux-Grues, c'est que, intuitivement au début, et plus tard consciemment, les insulaires ont développé un mode de vie ayant permis l'exploitation de la batture à des fins économiques locales et régionales, et ce, sans la détruire. Voilà un cas historiquement documenté de développement et d'exploitation durables.

Le problème aujourd'hui est que dans la plupart des études environnementales sur les terres humides (comme d'autres milieux, d'ailleurs), l'être humain est trop souvent perçu comme un agent ahistorique (c'est-à-dire une variable statique) et destructeur ${ }^{43}$. Dans le cas de l'Isle-aux-Grues, c'est tout le contraire qui s'est produit. Comme l'ont montré les données historiques et géographiques, il est possible de comprendre comment les êtres humains ont pu entretenir une relation à long terme avec un environnement sans y causer de dommages. Il est également possible de constater, comme plusieurs historiens de l'environnement (tels Cronon dans Changes in the Land, Judd dans Common Lands, Common People, ainsi que Park dans Ngã uruora) l'ont montré, que chaque dimension du passé, qui est très complexe, doit être étudiée (deep history), et que les activités humaines ne sont pas toujours nuisibles à l'environnement; elles peuvent au contraire être aussi très vulnérables aux changements socio-économiques ${ }^{44}$.

Selon les époques et les contextes socio-économiques, le type d'interaction avec le haut marais a varié. Les Amérindiens ont pratiqué la chasse, la pêche et la cueillette, et les colons, devenus insulaires, y ont ajouté la récolte du foin et le pâturage. Aujourd'hui, en contexte

43. J. Gauthier, D. Lehoux et J. Rosa, Les marécages intertidaux..., op. cit., 1; Ursula Larouche et Richard Legault, Guide des milieux humides du Québec (Charlesbourg, L’Union québécoise pour la conservation de la nature, 1993).

44. W. Cronon, Changes in the Land..., op. cit.; Richard W. Judd, Common Lands, Common People: The Origins of Conservation in Northern New England (Cambridge, Harvard University Press, 1997); G. Park, Ngã Uruora-The Groves of Life..., op. cit. 
postindustriel, on peut voir que les activités liées au haut marais (chasse, ornithologie, tourisme de plein air et récolte de foin pour la production de fromage) s'orientent vers des valeurs beaucoup plus sensibles aux questions environnementales. À une époque où les sociétés québécoise, canadienne et mondiale recherchent des façons de mieux intégrer les activités humaines et l'environnement, on a là un exemple de relation durable entre la société et la nature ${ }^{45}$. Sans prétendre à l'universalité de ses enseignements, on pourrait peut-être y trouver des principes applicables à l'étude, voire à l'aménagement, d’autres milieux.

45. Eric Higgs, Nature By Design: People, Natural Processes, and Ecological Restoration (Cambridge, The MIT Press, 2003); Christopher B. Barrett et Ray Grizzle, «A Holistic Approach to Sustainability Based on Pluralism Stewardship ", Environmental Ethics, 21 (1999): 23-42. 